http://dx.doi.org/10.21611/qirt.1992.041

\title{
Numerical reconstruction of interfacial defects and interface thermal resistances using thermal measurements
}

\author{
by M. BONNET, H. MAIGRE and M. MANAA
}

Laboratoire de Mécanique des Solides, URA CNRS 317, Ecole Polytechnique, 91128 Palaiseau Cedex, France.

\begin{abstract}
Thermography provides an experimental mean to control the quality of interface in laminates or in coatings. It is thus important to link thermal measurements obtained on free surfaces to the possible interfacial defects. In this paper a numerical inverse method is proposed to localize and quantify the defects from measured thermal data. The identification problem leads to a non-linear minimization problem solved using gradient methods.
\end{abstract}

\section{Introduction}

In elaboration of fiber composites or coatings, a very important point is the realisation of a perfect interface between the fiber and the matrix or between the coating and its substrate. Some interfacial defects may appear like delamination or like presence of undesirable matter between layers of the laminates. It is very important to localize such defects and also to determine their intensity (quality of the interface). As defects are located inside the materials, they cannot be observed or detected directly but it is already possible to obtain qualitative information from measurements done on free surfaces away from the interface. We present in this paper a numerical method to identify and quantify interfacial defects in materials using thermal data usually obtained using non destructive testing methods like infrared thermography.

\section{Modelisation of interfacial defects}

The problem adressed here is a two layer material as shown figure 1. The boundary surface $S_{m}$ is plane and infinite as well as the interface $S_{i}$ located at a known depth. Moreover, the second layer extends to infinity in depth. Infrared thermography is a means to detect possible interfacial defects. To do so, a known thermal flux is applied on the surface $S_{m}$ and in the same time, a thermal detector measures the temperature on this surface. The modelling of this problem is indicated on figure 2.. The two homogeneous layers occupy respectively the geometrical domains $\Omega_{1}$ and $\Omega_{2}$. For each domain $\Omega_{n}$, the thermal properties are the specific heat $c_{n}$, the density $\rho_{n}$ and the thermal conductivity $k_{n}$. To model the interface and its defects, we define an interface thermal resistance $R(x)$, that is, the temperature jump is proportional to the heat flux $q(x, t)$ across $S_{i}$ : 


$$
\|T\|(x, t)=-R(x) q(x, t)
$$

where

$$
q(x, t)=-k_{ \pm} \frac{\partial T}{\partial y}\left(x, h_{ \pm}, t\right)
$$

$T(x, y, t)$ is the temperature field and $\|\cdot\|$ denotes the jump operator across $S_{i}$ : $\|f\|(x)=f\left(x, h_{2}, t\right)-f\left(x, h_{1}, t\right)$.

Besides, the temperature evolution in the conductive medium is governed by the wellknown homogeneous transient heat conduction equation:

$$
\operatorname{div}\left(k_{n} \nabla T\right)-\rho_{n} c_{n} \frac{\partial T}{\partial t}=0 \quad n=1,2
$$

The known thermal flux applied on $S_{m}$ is:

$$
g(x, t)=-k_{1} \frac{\partial T}{\partial y}(x, t)
$$

and as in any transient problem we need to precise also the initial conditions which are:

$$
T(x, y, 0)=0 \quad \text { in } \Omega_{1} \cup \Omega_{2}
$$

\section{Identification problem}

The identification problem consists in determining the interface resistance $R(x)$ from the measurable data e.g. the temperature $T_{m}(x, t)$ for $x \in S_{m}$ and $t \in[0, \tau] . \tau$ represents the duration of the measurements.

If $T_{R}(x, y, t)$ denotes the temperature field governed by equations (1), (2), (3) and (4), one can remark that the temperature measurement on $S_{m}$ acts as a overspecified data and allows one to reconstruct the interface resistance $R(x)$. To do so, $R(x)$ is treated as a minimizer of the least-squares functional $J(R)$ :

$$
J(R)=\frac{1}{2} \int_{0}^{\tau} \int_{S_{m}}\left[T(x, t)-T_{m}(x, t)\right]^{2} d S d t
$$

under the constraint

$$
T(x, t)=T_{R}(x, 0, t) \quad \text { in } S_{m} \times[0, \tau]
$$

Hence the reconstruction problem leads to a non-linear constrained least-squares problem.

\section{Calculation of the gradient for the minimization}

As the minimization problem has no explicit solution, a way to minimize the functional $J$ is to use non-linear optimisation algorithms. The most efficient ones, e.g. conjugate gradient or BFGS variable metric [1], need not only $J(R)$ but also its gradient with respect to the design variable $R$. The latter could be computed using finite difference 
formulae, but it is far safer, from the point of view of accurancy and efficiency, to use an exact expression of the gradient.

However, differentiation of (5) is by no means a trivial matter, since $J$ depends functionally on $R$.

Let the set $V$ of scalar functions $v(x, y, t) \quad\left\{(x, y) \in \Omega_{1} \cup \Omega_{2} ; t \in[0, \tau]\right\}$ be defined as:

$$
V=\left\{v(x, y, t), v \in L^{2}, \nabla v \in L^{2}, v(x, y, 0)=0\right\}
$$

The temperature field $T_{R}$ solves the following variational formulation, which is equivalent to equations (1), (2), (3) and (4); find $T \in V$ such that:

$$
\forall w \in V \quad \int_{\Omega}\left\{k \nabla T \cdot \nabla w+\frac{\partial T}{\partial t} w\right\} d \Omega=\int_{S_{m}} g w d S+\int_{S_{i}} \frac{\|T\|\|w\|}{R} d S
$$

In order to express the gradient of the functional $J$ under the constraint (6), let us introduce the Lagrangian $L$ :

$$
\begin{gathered}
\mathcal{L}(T, w, R)=\int_{0}^{\tau} \int \frac{1}{2} \int_{S_{m}}\left(T-T_{m}\right)^{2} d S+\int_{S_{m}} g w d S+\int_{S_{i}} \frac{\|T\|\|w\|}{R} d S \\
\left.-\int_{\Omega}\left(k \nabla T \cdot \nabla w+\frac{\partial T}{\partial t} w\right) d \Omega\right\} d t
\end{gathered}
$$

One has evidently $J(R)=L(T, w, R)$ if the constraint (6) is satisfied. The gradient of $J$ will thus be investigated by means of the gradient of $L$. As $L$ is a functional, we introduce an evolution of the functions $T, w, R$ defined by the parameter $\eta$ and the pseudovelocities $\dot{T}, \dot{W}, \dot{R}$ :

$$
T(\eta)=T+\eta \dot{T} \quad \cdot \quad w(\eta)=w+\eta \dot{w} \quad R(\eta)=R+\eta \dot{R}
$$

Then the derivative $\partial L / \partial \eta$ of the functional $L$ considered as a function of $\eta$ is a linear form of the functions $\dot{T}, \dot{w}, \dot{R}$. In the following, all derivatives with respect to $\eta$ will be implicitely taken for $\eta=0$. The result is a sum of three linear forms as follows:

$$
\begin{aligned}
& \frac{\partial L}{\partial \eta}=\mathcal{L}_{, T}(\dot{T})+\mathcal{L}_{, w}(\dot{w})+\mathcal{L}_{, R}(\dot{R}) \\
& \mathcal{L}_{, T}(\dot{T})=\int_{0}^{\tau}\left\{\int_{S_{m}}\left(T-T_{m}\right) \dot{T} d S+\int_{S_{i}} \frac{\|\dot{T}\|\|w\|}{R} d S-\int_{\Omega}\left(k \nabla \dot{T} \cdot \nabla w+\frac{\partial \dot{T}}{\partial t} w\right) d \Omega\right\} d t \\
& \mathcal{L}_{, W}(\dot{w})=\int_{0}^{\tau}\left\{\int_{S_{m}} g \dot{w} d S+\int_{S_{i}} \frac{\|T\|\|\dot{w}\|}{R} d S-\int_{\Omega}\left(k \nabla T \cdot \nabla \dot{w}+\frac{\partial T}{\partial t} \dot{w}\right) d \Omega\right\} d t \\
& \mathcal{L}_{, R}(\dot{R})=-\int_{0}^{\tau} \int_{S_{I}} \frac{\|T\|\|w\|_{\dot{R}}}{R^{2}} d S d t
\end{aligned}
$$

where $L_{, f}$ represents the linear tangent application with respect to the field $f$. The gradient of $J$ is also determined by its linear form of the function $\dot{R}$ : 


$$
\frac{\partial J}{\partial \eta}=J_{, R}(\dot{R})
$$

and the important result is:

$$
\begin{aligned}
& \text { if } \quad\left\{\begin{array}{l}
L_{, T}=0 \\
L_{, w}=0
\end{array}\right. \text { then } \\
& \frac{\partial J}{\partial \eta}=\frac{\partial \mathcal{L}}{\partial \eta} \quad \text { or } \quad J_{, R}=L_{, R}
\end{aligned}
$$

The second condition of (13) is obtained when $T$ is the solution of the problem governed by equations. (1), (2), (3) and (4). The first condition of (13) gives a variational equation on $w$ and its solution, $w_{H}$, is called the adjoint state. A detailed examination of this equation shows that $w$ is equivalently governed by the following final-boundary value problem:

$$
\begin{aligned}
& \|w\|(x, t)=-R(x) q(x, t) \quad \text { where } \quad q(x, t)=-k_{ \pm} \frac{\partial w}{\partial y}\left(x, h_{ \pm}, t\right) \\
& \operatorname{div}\left(k_{n} \nabla w\right)+\rho c_{n} \frac{\partial w}{\partial t}=0 \quad n=1,2 \\
& k_{1} \frac{\partial w}{\partial y}(x, 0, t)=-\left[T(x, 0, t)-T_{m}(x, t)\right] \text { on } S_{m} \\
& T(x, y, \tau)=0 \quad \text { in } \Omega_{1} \cup \Omega_{2}
\end{aligned}
$$

It is apparent in (16) that $w$ is governed by the backward heat equation: the evolution problem for $w$ must be solved from $t=\tau$ to $t=0$.

\section{Numerical identification}

\subsection{Resolution procedure}

To realize the identification of the interface resistance $R$, we propose to use a transient thermal code which can take into account jumps of temperature at the interface. The minimisation of $J$ is performed by a succession of iterations. Each iteration, in which $R$ is fixed, consists in four steps:

- direct computation of the temperature $T$ following the equations (1), (2), (3), and (4);

- reverse computation of the adjoint state $w$ from equations (15), (16), (17), and (18);

- computation of $J_{, R}$ from equations (12) and (14);

- modification of $R$ which leads to a decrease of $J$.

One must remark that the computation of the adjoint state $w$ can be done with the same code that computes the temperature $T$ if the following transformation is respected:

$$
w(x, y, t)=\omega(x, y, \tau-t)
$$




\section{http://dx.doi.org/10.21611/qirt.1992.041}

\section{2. $1 \mathrm{D}$ results}

A first investigation was done on a 1D model to test the identification method using the calculation of the gradient of $J$. All the thermal fields were assumed to be uniform along the $x$-axis and to depend only on $y$ and $t$. Simulated data with a known resistance $R$ were used for the imposed flux $g$ and for the measured temperature $T_{m}$. Several cases were studied depending on the conductivity of the two layers, on the simulated resistance and on the noise added to the measured temperature (see table 1). To indicate the effects of noise, the reconstructed value of $R$ is given within a range corresponding to twice the value of $J_{\min }$. With no noise the reconstruction is always perfect whereas the presence of noise leads to inaccurate values especially if the duration of the measurements is short.

\section{Conclusions}

The detection of internal interfacial defects found in laminates or coatings can be performed with the identification method presented above based on a minimization problem. This numerical method is all the more efficient since it quantifies the defects through the thermal resistance $R$ and allows the use of optimization algorithms with gradient. We propose an exact expression of the gradient which needs no more computation than a classical transient thermal problem.

This method of defect identification may be generalized to more complicated geometries and to other boundary conditions. It is also possible to consider the depth location of the interface as an unknown function and this leads to the calculation of the gradient of $J$ with respect to a surface location.

Futhermore, the inverse problem decripted above is ill-posed [2] due to the nature of the integral operators involved [3] and due to unavoidable noise of the measured data. As a consequence, the procedure needs to be regularized in order to stabilize its solution and avoid erroneous results caused by noised data. The regularization consists in introducing a stabilization functional $S(R)$ which incorporates a priori informations like physically relevant range of values, rough estimate of the location of defects, expected regularities...

\section{REFERENCES}

[1] PRESS (W.H.), FLANERY (B.P.), TEUKOLSKY (S.A.) and VETTERLING (W.T.). Numerical recipes: the art of scientific computing. Cambridge Press, 1986.

[2] TIKHONOV (A.N.) and ARSENIN (V.Y.). - Solutions of ill-posed problems. WinstonWiley, New-York, 1977.

[3] BONNET (M.), BUI (H.D.) and PLANCHARD (J.). - Problèmes inverses pour l'équation de la chaleur: application au contrôle non destructif thermique. Note interne EDF/DER, 1989.

[4] BONNET (M.). - Identification et contrôle par équations intégrales de frontière et variation de domaine. GRECO Calcul des Structures, in Calcul des structures et intelligence artificielle, vol.4, ed. Pluralis, 1990, p. 25-36. 
http://dx.doi.org/10.21611/qirt.1992.041

[5] BONNET (M.), BUI (H.D.), MAIGRE (H.) and PLANCHARD (J.) - Identification of heat conduction coefficient : application to non destructive testing. IUTAM Symposium on Inverse problems in engineering mechanics, 11-15 May 1992, Tokyo, Japan.

\begin{tabular}{|c|c|c|c|c|c|c|}
\hline \multicolumn{7}{|c|}{ Table 1. - Results of a 1D example. } \\
\hline 12 & $k_{1}$ & $k_{2}$ & Simulated $R$ & Noise 0\% & Noise 1\% & Noise 3\% \\
\hline 12 & 10 & 1 & 1 & $\begin{array}{c}1 \\
1-1\end{array}$ & $0.84-1.14$ & $0.54-1.49$ \\
\hline 12 & 1 & 10 & 1 & $1-1$ & $0.94-1.05$ & $0.83-1.19$ \\
\hline 12 & 1 & 1 & 3 & $1-1$ & $0.92-1.06$ & $0.75-1.23$ \\
\hline 6 & 1 & 1 & 1 & $1-3$ & $2.46-3.47$ & $1.7-4.9$ \\
\hline 4 & 1 & 1 & 1 & 1 & 1.02 & \\
& & & & $1-1$ & $0.16-6.5$ & \\
\hline
\end{tabular}

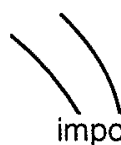

imposed

heat flux

15

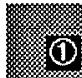

(2)

1):
Interfacial defects

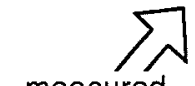

measured

temperature

$s_{m}$

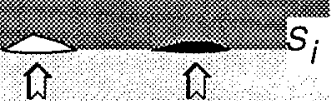

$S_{i}$

$\Leftrightarrow$

\section{imposed measured \\ heat flux temperature}

$g(x, t) \quad T_{m}(x, t) \quad S_{m}$

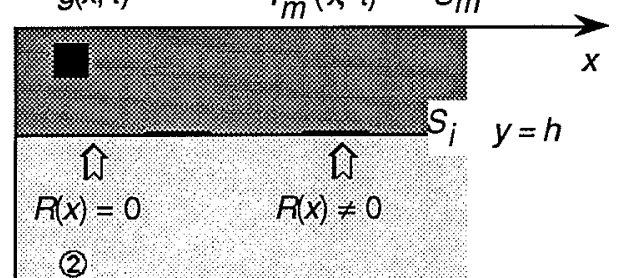

$\checkmark y$
Fig. 1. - Thermography on a laminate with interfacial defects.
Fig. 2. - Modelisation of the interfacial defects. 